\title{
Fatores associados às alterações na velocidade de marcha e força de preensão manual em idosos institucionalizados
}

\section{Factors assodiated withalterationsin thegait speed and handgipininstitutionalizededderly}

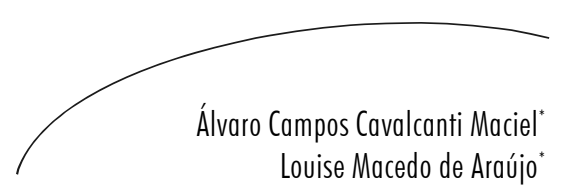

Objetivo: Este trabalho teve como propósito avaliar os fatores associados às alterações na velocidade de marcha e força de preensão manual em idosos institucionalizados da cidade de Natal-RN. Metodologia: Trata-se de estudo transversal, realizado com uma amostra de 20 idosos (11 mulheres e 9 homens), selecionados de forma não aleatória por conveniência. Os dados foram obtidos através de questionário e mensurações da força de preensão manual e velocidade de marcha, que constituíram os testes físicos. Resultados: Foram encontradas diferenças estatisticamente significativas $(\mathrm{p}<0,05)$ entre os sexos quanto ao tempo de institucionalização, sensação de fadiga autorrelatada, força de preensão e nível cognitivo. Também foram verificadas correlações entre a força de preensão manual com o tempo de asilo $(r=$ $-0,54)$, função cognitiva (MEEM) $(r=0,62)$, altura $(r=0,79)$ e massa corporal $(r=$ $0,82)$; para a velocidade de marcha, houve correlação significativa com o MEEM $(\mathrm{r}=0,45)$ e independência nas atividades instrumentais da vida diária (AIVDs) ( $\mathrm{r}=$ 0,47). Conclusão: Alterações na força de preensão e velocidade de marcha são comuns entre os idosos institucionalizados, mais susceptíveis à síndrome da fragilidade e, portanto, a perdas funcionais e cognitivas, que favorecem a progressão de suas limitações. Desta forma, a investigação das condições de saúde desses indivíduos pode ser útil para detectar um perfil de fragilidade e identificar que tipo de cuidados e intervenções seriam prioritários para melhorar a funcionalidade e qualidade de vida destes idosos.

\section{Abstract}

Objective: This study aimed to evaluate the factors associated with changes in gait speed and strength of handgrip in institutionalized elderly in the city of Natal-RN. Methods: This is a cross-sectional study conducted with a sample of 20 elderly (11 women and 9 men), selected among a non-random for convenience. Data were collected by questionnaire and measurements of handgrip and gait speed, which constituted the physical tests. Results: We found statistically significant differences
Palavras-chave: Saúde do Idoso Institucionalizado. Força da Mão. Marcha. Atividades Cotidianas. Cognição. Força de Preensão Manual. NatalRN. 
$(p<0.05)$ between genders as to the time of institutionalization, a feeling of self reported fatigue, grip strength and cognitive level. We also found correlations between the handgrip and the time of asylum $(r=-0,54)$, cognitive function (MMSE) $(r=0,62)$, height $(r=0,79)$ and body mass $(r=0,82)$; gait speed was significantly correlated with MMSE $(\mathrm{r}=0,45)$ and independence in instrumental activities of daily living (IADLs) $(r=0,47)$. Conclusion: Changes in handgrip and gait speed are common among the institutionalized elderly, more susceptible to the syndrome of frailty, and thus the functional and cognitive losses, which favor the advancement of their limitations. Thus, investigating the health of these individuals may be useful to detect a profile of vulnerability and identify what type of care and intervention priority would be to improve to increase the functionality and quality of life of these elderly.
Key words: Health of Institutionalized Elderly. Hand Strength. Gait. Activities of Daily Living. Cognition. Handgrip. Natal city.

\section{INTRODUÇC̃̃O}

O processo de envelhecimento vem exercendo substancial influência sobre o desenvolvimento e funcionamento das sociedades, de tal forma que todos os países tendem a incluir as possíveis repercussões desse acontecimento como assuntos prioritários no âmbito da saúde pública e da economia. ${ }^{1}$ Este fenômeno mundial tem levado a uma reorganização do sistema de saúde, pois essa população exigecuidados que são um desafio, devido às doenças crônicas que apresentam, além do fato de que incorporam disfunções nesta etapa da vida. ${ }^{2}$

De acordo com Lima-Costa \& Veras, ${ }^{3}$ as enfermidades presentes no envelhecimento são condicionadas pela vulnerabilidade do avanço da idade e pelos fatores de risco variados, sendo, portanto, multicausais, e produzindo polipatologias sobre os diversos sistemas e funções.

Garrido \& Menezes ${ }^{1}$ relatam também que a cronicidade com que cursa a maioria das doenças origina limitações orgânicas e funcionais que impedem os idosos realizarem, de forma progressiva, atividades próprias da vida diária (AVDs), o que os obriga a buscarem ajuda mediante diferentes recursos: técnicos, materiais e/ou pessoais. Neste sentido, o idoso se configura como um indivíduo totalmente passível de ser acometido por algum tipo de patologia, tanto física quanto mental ou social, colocando-se em situação de fragilidade que o torna mais vulnerável a qualquer agente agressor.

$\mathrm{Na}$ busca de uma medida mais adequada para mensurar as manifestações patológicas do envelhecimento, geriatras e gerontólogos vêm utilizando o termo "fragilidade" para caracterizar idosos mais debilitados e vulneráveis. ${ }^{4,5}$

Atualmente, o conceito mais plausível para definir fragilidade é proposto por Fried et al., ${ }^{4,6}$ que a definem como uma síndrome de declínio espiral de energia, embasada por alterações relacionadas ao envelhecimento, composto principalmente por sarcopenia, distúrbios neuroendócrinos e disfunção imunológica. A sarcopenia, condição altamente prevalente em idosos, definida como declínio progressivo da massa e força muscular, tem grande impacto na capacidade funcional desses indivíduos, pois, sendo importante preditor da fragilidade, traz como consequências, além da diminuição da força muscular, baixa tolerância ao exercício e redução da velocidade de marcha. ${ }^{7}$

Dentre os critérios que caracterizam o fenótipo da fragilidade, merecem destaque a redução da velocidade de marcha e a diminuição da força de preensão manual, que é bastante utilizada como indicador de força muscular geral por ser um parâmetro fácil de medir. $\mathrm{O}$ enfoque nestes critérios se deve ao fato de que estas alterações conferem maior risco para dependência nas atividades básicas ou instrumentais da vida diária, para a incapacidade, quedas, fraturas, hospitalização recorrente e morte. ${ }^{7}$

O quadro acima citado torna-se ainda mais grave com o processo de institucionalização, sendo os idosos asilados, na maioria das vezes, mais frágeis. ${ }^{8,9}$ Neste caso, além das limitações 
funcionais que levam a restrições na independência e autonomia, notadamente marcadas pela dificuldade ao realizar as AVDs, os idosos apresentam exacerbação dos déficits cognitivos. ${ }^{10}$

As alterações cognitivas envolvem as faculdades intelectuais, memória, atenção, orientação espaçotemporal e a capacidade de realizar tarefas cotidianas. Diante desta gama de alterações, surgem limitações físicas e funcionais com progressão para incapacidades definitivas, que por sua vez podem evoluir para o óbito, já que esses indivíduos passam a avaliar de modo irrealista suas capacidades e perdas, podendo subestimar os riscos envolvidos em certas atividades. ${ }^{11}$

A associação de vários fatores limitantes somase ao fato de os idosos asilados normalmente viverem em um ambiente despreparado para atender suas necessidades, no que diz respeito a promoção de lazer, socialização e saúde, condicionando-os ao isolamento e inatividade. Esses aspectos, não raras vezes, evoluem para um quadro de invalidez, depressão e demência, favorecendo o surgimento ou agravamento da fragilidade e trazendo impactos socioeconômicos de extrema relevância. ${ }^{12}$

Diante deste paradigma, torna-se imprescindível a preservação e/ou recuperação da capacidade funcional do idoso, em que a fisioterapia tem papel fundamental, pois através da educação em saúde, exercícios, recursos e técnicas específicas, o fisioterapeuta consegue promover o adiamento da instalação das incapacidades, já que contribui para a melhora da força, mobilidade, equilíbrio, realização das AVDs, redução do número de quedas e, consequentemente, para o bem-estar mental e qualidade de vida. ${ }^{8,13}$

Levando em consideração as alterações funcionais preditoras da síndrome da fragilidade e os agravos decorrentes da institucionalização, este trabalho tem como objetivo avaliar os fatores associados às alterações na velocidade de marcha e força de preensão manual em idosos institucionalizados.

\section{METODOLOGIA}

Trata-se de estudo observacional analítico, de caráter transversal, realizado numa instituição asilar da cidade de Natal-RN. A instituição conta com 187 residentes, sendo 130 mulheres e 57 homens.

Os seguintes parâmetros estatísticos foram adotados no cálculo do tamanho amostral: erro estatístico máximo de 5,0\% para o tipo I e 20,0\% para o erro de tipo II, com um poder de $80,0 \%$ para o estudo. Para um desvio de $5,0 \%$, para mais ou para menos, nas estimativas efetuadas e, considerando a população de referência em 187 idosos com 65 anos ou mais, em 2009 foi definida uma amostra de 20 indivíduos ( $11 \%$ da população inicial). Deste modo, 20 indivíduos, sendo 11 mulheres e 9 homens, foram selecionados de forma não-aleatória por conveniência, a partir da escolha dos idosos em condições cognitivas de responder ao questionário. Para a possível identificação da amostra, foram contatados os responsáveis pelo asilo, os quais assinaram um termo de autorização para realização do estudo. Da mesma forma, informaram quais idosos estariam em condições mentais de participar do estudo.

O critério de exclusão adotado foi a presença de déficit cognitivo, medido pelo Mini-Exame do Estado Mental (MEEM) ${ }^{14}$ ou alguma situação clínica que impedisse o idoso de responder ao questionário, bem como executar os testes físicos, como por exemplo, déficit cognitivo grave e dependência de terceiros para deambular.

A avaliação dos idosos foi realizada na própria instituição asilar, através de entrevistas e avaliação instrumental da força de preensão manual, utilizando um dinamômetro manual e análise da velocidade de marcha.

As variáveis independentes foram divididas em quatro grupos:

- Variáveis sócio-demográficas: idade, tempo de asilo e escolaridade. 
- Variáveis de saúde física: doenças crônicas autorrelatadas ${ }^{15}$ (doença do coração, hipertensão, diabetes mellitus, artrite ou reumatismo, depressão e osteoporose); autopercepção da saúde; massa corporal e altura.

- Variáveis de capacidade funcional: escore de independência para AIVDs, em que foi avaliada a total independência do idoso no que se refere à realização das atividades instrumentais da vida diária (AIVDs), que compreendem tarefas como usar o telefone, usar o transporte, fazer compras, preparo da alimentação, tarefas domésticas, manejo de medicação e de dinheiro, como proposto pela escala desenvolvida por Lawton \& Brody, ${ }^{16}$ escore de independência para ABVDs, em que se utilizou o Índice de Katz ${ }^{17}$ para avaliar a total independência em relação às atividades básicas da vida diária (ABVDs). Este índice conta com os seguintes itens: alimentação, higiene pessoal, onde se incluem o banho e o uso do vaso sanitário, vestir-se, transferências, deambulação e controle dos esfíncteres. Os escores para estas variáveis foram determinados pela soma das atividades que eram realizadas com total independência pelos idosos participantes. Por fim, a fadiga foi avaliada por autorrelato, de acordo com os itens 7 (Sentiu que teve que fazer esforço para dar conta das suas tarefas habituais?) e 20 (Não conseguiu levar adiante as suas coisas?) da escala para rastreamento da depressão CES-D. ${ }^{18}$ Estes itens foram representados, respectivamente, pelas variáveis fadiga 1 e fadiga 2, mensuradas com base num escore que podia variar de 1 a 4, em que o maior valor corresponderia à maior sensação de fadiga.

- Variável neuropsíquica: escore do MiniExame do Estado Mental (MEEM). ${ }^{14}$ Foi utilizado o escore de 17 como ponto de corte; independentemente do grau de escolaridade (Brucki et al., 2003); ${ }^{19}$ esta escolha teve como objetivo evitar perder muitos idosos em decorrência de um ponto de corte muito alto e incluir idosos sem condições de compreender os comandos para os testes físicos, caso o ponto de corte fosse muito baixo.

As variáveis dependentes, por sua vez, foram a força de preensão manual e a velocidade de marcha.

Dentre os instrumentos utilizados, para a medida da massa corporal foi utilizada uma balança Filizona, Série 3.134, com divisões de 100g; para a tomada da altura, fitas métricas de "fiber glass" com divisões de $1 \mathrm{~mm}$, e a força de preensão manual foi determinada por um dinamômetro manual hidráulico JAMAR ${ }^{\circledR}$ modelo J00105.

Para a mensuração da força de preensão manual, os indivíduos foram posicionados sentados, com o braço aduzido e paralelo ao tronco, cotovelo fletido a $90^{\circ}$ e antebraço e punho em posição neutra. Foram realizadas três medições, no membro dominante, com intervalo mínimo de 30 segundos entre elas, e anotado o maior valor. Quanto à análise da velocidade de marcha, esta foi feita num percurso de 4,6 metros, em linha reta, com marcação do tempo em milésimos de segundos e, logo após, cálculo e registro da velocidade média alcançada.

Os dados deste trabalho foram coletados em março de 2009 e os entrevistadores foram devidamente treinados durante o estudo piloto prévio. Para o processamento, armazenamento e análise dos dados, foi utilizado o programa estatístico SPSS (Statistical Package for the Social Sciences), versão 13.0 .

A estatística descritiva foi realizada usando-se medidas de tendência central e dispersão (média, mediana e desvio padrão), resumidas na linha de base de acordo com o sexo. Para a verificação da normalidade dos dados, foi utilizado o teste de Kolmogorov-Smirnov (K-S). Em seguida, foi realizada análise de correlação de Pearson entre as variáveis força de preensão e velocidade da marcha com as variáveis independentes 
quantitativas, e teste $t$, para amostras não pareadas com as variáveis categóricas. Em toda a análise estatística, foi considerado um intervalo de confiança (IC) de 95\% e um p=0,05.

Todos os participantes foram previamente informados sobre o objetivo da pesquisa e procedimentos realizados e assinaram o Termo de Consentimento Livre e Esclarecido, como determinado pela Resolução no 196/96, do Conselho Nacional de Saúde (CNS) ${ }^{20}$

\section{RESULTADOS}

A amostra foi composta por $20 \mathrm{idosos}$ residentes numa instituição asilar da cidade de Natal-RN, sendo nove homens e 11 mulheres. Pelos dados apresentados de média e desvio padrão, a amostra apresentou distribuição normal.

As características da amostra quanto às variáveis sócio-demográficas, de saúde física, de capacidade funcional e neuropsíquica estão descritas na tabela 1.

Tabela 1 - Distribuição das variáveis independentes de acordo com o sexo. Natal, RN, 2009.

\begin{tabular}{llll}
\hline VARIÁVEIS & MASCULINO & FEMININO & TOTAL \\
\hline Idade (anos) & $76,2( \pm 4,5)$ & $79,4( \pm 4,7)$ & $78,0( \pm 4,8)$ \\
Tempo de asilo (anos) & $3,4( \pm 2,9)^{*}$ & $12,7( \pm 7,3)$ & $8,5( \pm 7,4)$ \\
Escolaridade (anos) & $3,2( \pm 1,1)$ & $2,4( \pm 0,9)$ & $3,6( \pm 2,8)$ \\
Cardiopatia (\%) & 33,0 & 18,3 & 25,0 \\
Hipertensão (\%) & 77,8 & 54,5 & 65,0 \\
Diabetes mellitus (\%) & 33,0 & 18,2 & 25,0 \\
Reumatismo (\%) & 44,4 & 45,5 & 45,0 \\
Depressão (\%) & 22,2 & 36,4 & 30,0 \\
Percepção da saúde (\%) & & & \\
Boa & 57,1 & 42,9 & 47,4 \\
Ruim & 41,7 & 58,3 & 52,6 \\
Escore de independência para AIVDs & $5,7( \pm 2,1)$ & $4,2( \pm 2,0)$ & $4,8( \pm 2,1)$ \\
Escore de independência para ABVDs & $5,9( \pm 0,3)$ & $5,6( \pm 0,6)$ & $5,8( \pm 0,4)$ \\
Fadiga 1 & $1,9( \pm 0,9)$ & $1,7( \pm 0,6)$ & $1,8( \pm 0,8)$ \\
Fadiga 2 & $1,1( \pm 0,3)^{*}$ & $1,5( \pm 0,5)$ & $1,4( \pm 0,5)$ \\
Massa corporal (kg) & $72,1( \pm 10,3)$ & $51,6( \pm 9,4)$ & $60,9( \pm 14,1)$ \\
Altura (cm) & $165,2( \pm 7,2) *$ & $148,2( \pm 8,1)$ & $1,6( \pm 0,1)$ \\
Força de preensão manual (kgf) & $27,9( \pm 5,9)^{*}$ & $15,2( \pm 4,6)$ & $20,9( \pm 8,0)$ \\
Velocidade da marcha (m/s) & $0,8( \pm 0,4)$ & $0,7( \pm 0,2)$ & $0,7( \pm 0,3)$ \\
Escore do MEEM & $24,1( \pm 3,2)^{*}$ & $19,8( \pm 1,9)$ & $18,5( \pm 7,4)$ \\
\hline
\end{tabular}

$* p>0,05$

Os valores das correlações entre as variáveis independentes quantitativas com a força de preensão manual e com a velocidade de marcha estão apresentados na tabela 2. Para a força de preensão manual, obtiveram-se correlações estatisticamente significativas com o tempo de asilo dos idosos participantes, com a função cognitiva (MEEM), com a altura e massa corporal. Em relação à velocidade de marcha, houve correlação significativa com a função cognitiva e com as atividades instrumentais da vida diária (AIVDs). 
Tabela 2 - Correlação entre as variáveis independentes e dependentes. Natal, RN, 2009.

\begin{tabular}{lcc}
\hline VARIÁVEIS & FORÇA DE PREENSÃO & VELOCIDADE DA MARCHA \\
\hline Idade & $\mathrm{r}=-0,28 ; \mathrm{p}=0,22$ & $\mathrm{r}=-0,31 ; \mathrm{p}=0,17$ \\
Tempo de asilo & $\mathrm{r}=-0,54 ; \mathrm{p}=0,01$ & $\mathrm{r}=-0,21 ; \mathrm{p}=0,39$ \\
Escolaridade & $\mathrm{r}=0,39 ; \mathrm{p}=0,08$ & $\mathrm{r}=0,19 ; \mathrm{p}=0,41$ \\
MEEM & $\mathrm{r}=0,62 ; \mathrm{p}=0,03$ & $\mathrm{r}=0,45 ; \mathrm{p}=0,04$ \\
ABVDs & $\mathrm{r}=0,39 ; \mathrm{p}=0,08$ & $\mathrm{r}=0,12 ; \mathrm{p}=0,64$ \\
AIVDs & $\mathrm{r}=0,58 ; \mathrm{p}=0,07$ & $\mathrm{r}=0,47 ; \mathrm{p}=0,03$ \\
Fadiga 1 & $\mathrm{r}=-0,04 ; \mathrm{p}=0,8$ & $\mathrm{r}=0,07 ; \mathrm{p}=0,7$ \\
Fadiga 2 & $\mathrm{r}=-0,14 ; \mathrm{p}=0,14$ & $\mathrm{r}=-0,34 ; \mathrm{p}=0,13$ \\
Altura & $\mathrm{r}=0,79 ; \mathrm{p}<0,001$ & $\mathrm{r}=-0,39 ; \mathrm{p}=0,18$ \\
Massa corporal & $\mathrm{r}=0,82 ; \mathrm{p}<0,001$ & $\mathrm{r}=-0,35 ; \mathrm{p}=0,12$ \\
\hline
\end{tabular}

Os dados que são apresentados na tabela 3 referem-se às médias da velocidade de marcha e da força de preensão manual em relação às doenças crônicas autorrelatadas. Não houve diferença nas médias das variáveis dependentes em nenhuma das doenças avaliadas.

Tabela 3 - Médias dos valores de velocidade de marcha e força de preensão manual em relação às doenças autorrelatadas. Natal, RS, Brasil. 2009.

\begin{tabular}{lcc}
\hline VARIÁVEL & $\begin{array}{c}\text { VELOCIDADE DE } \\
\text { MARCHA }\end{array}$ & $\begin{array}{c}\text { FORÇA DE PREENSÃO } \\
\text { MANUAL }\end{array}$ \\
\hline Doença do coração & $0,82( \pm 0,26)$ & $24,93( \pm 7,82)$ \\
Sim & $0,71( \pm 0,31)$ & $19,60( \pm 7,92)$ \\
Não & $0,82( \pm 0,31)$ & $21,95( \pm 8,30)$ \\
Hipertensão & $19,04( \pm 7,79)$ \\
Sim & $0,59( \pm 0,23)$ & \\
Não & $0,73( \pm 0,38)$ & $22,40( \pm 7,37)$ \\
Diabetes mellitus & $0,74( \pm 0,28)$ & $20,44( \pm 8,45)$ \\
Sim & & \\
Não & $0,79( \pm 0,28)$ & $21,70( \pm 7,29)$ \\
Artrite/Reumatismo & $0,70( \pm 0,32)$ & $20,30( \pm 8,92)$ \\
Sim & & \\
Não & $0,79( \pm 0,30)$ & $22,66( \pm 7,61)$ \\
Depressão & $20,19( \pm 8,39)$ \\
Sim & $0,72( \pm 0,31)$ & \\
Não & & $16,99( \pm 5,18)$ \\
Osteoporose & $0,60( \pm 0,14)$ & $21,37( \pm 8,29)$ \\
Sim & $0,76( \pm 0,31)$ & \\
Não & &
\end{tabular}




\section{DISCUSSÃO}

O objetivo deste estudo foi identificar a relação existente entre os fatores sócio-demograficos, de saúde física, de capacidade funcional, neuropsíquicos e antropométricos com as alterações na velocidade de marcha e força de preensão manual numa coorte de idosos institucionalizados.

Pelos achados desta pesquisa, observou-se diferença significativa entre os sexos no que diz respeito ao tempo de institucionalização, sendo o das mulheres maior do que o dos homens. Pavan et al. ${ }^{21}$ apontam o sexo feminino como uma condição a mais de vulnerabilidade para a institucionalização, pois, além de as mulheres viverem mais do que os homens, experimentam uma probabilidade maior de ficarem viúvas mais cedo, passando a viverem sozinhas, já que apresentam dificuldades para casar novamente. Além disso, possuem menor nível de escolaridade e, consequentemente, status social inferior, tendendo a condições econômicas desvantajosas. Surge, então, um fator preocupante, tendo em vista que quando ficam mais velhas, desenvolvem necessidades especiais e maior risco de dependência, precisando de recursos financeiros para os cuidados com a saúde e melhor qualidade de vida. ${ }^{22}$ Diante disto, muitas vezes o processo de institucionalização é encarado como única opção.

Com relação à fadiga, os homens apresentaram menor sensação autorrelatada do que as mulheres, verificado por um menor escore em relação ao item 20 da escala CES-D. Segundo o estudo realizado por Valentine et al. ${ }^{23}$ com idosos sedentários e saudáveis, ficaram demonstradas diferenças entre os sexos na percepção de fadiga, mais relatada pelas mulheres do que pelos homens. Tal estudo identificou que o aumento da taxa de gordura, as inflamações sistêmicas verificadas pelo alto nível de proteína $\mathrm{C}$ reativa (PCR), a diminuição da aptidão física e a depressão são preditores independentes de fadiga para o sexo feminino; já para os homens, apenas a depressão foi um preditor independente. Uma explicação plausível é que a fadiga, critério relevante para a síndrome da fragilidade, leva a um menor nível de atividade física e, consequentemente, menor gasto energético, favorecendo o aumento da taxa de gordura corporal, mais inflamações e disfunções crônicas, que potencializam a fadiga autorreferida entre os idosos.

Bautmans et al. ${ }^{24}$ relatam que, devido à sarcopenia, os idosos desempenham suas funções num patamar próximo do seu limite de força máxima, pois as atividades diárias que realizam exigem muitas vezes contrações musculares intensas e sustentadas, as quais, diante da redução de força muscular nesses indivíduos, podem explicar a sensação comum de fadiga. Na mesma linha, Moreh et al. ${ }^{25}$ abordam a relação existente entre a sensação de fadiga e a declínio da funcionalidade, seja no âmbito da dependência ou na diminuição do desempenho das atividades diárias. Esses aspectos influenciam uma pior percepção da saúde e no aparecimento de comorbidades em idosos.

Diante de tantas implicações negativas na saúde do idoso, este preditor da fragilidade, responsável pela sensação constante de indisposição e cansaço, é também um preditor de mortalidade, observando-se maior índice de morte nos idosos com mais de 65 anos e com sintomas de fadiga do que naqueles que não estão dentro deste grupo. ${ }^{26}$

A força de preensão manual, por sua vez, foi maior no sexo masculino, o que talvez se explique pela maior massa corporal, melhor percepção da saúde, melhor nível funcional e cognitivo por parte dos homens avaliados. Dey et al. ${ }^{27}$ afirmam que é incerto que o declínio de força muscular na terceira idade seja atribuído somente à diminuição de massa muscular. Relatam que, além da perda progressiva de massa, surgem alterações estruturais músculo-esqueléticas que influenciam negativamente na função muscular. Pelos seus resultados, houve diminuição de massa em ambos os sexos e aumento do percentual de gordura apenas nos homens, repercutindo num declínio de força entre os idosos avaliados. No entanto, esse declínio ocorreu de modo mais proeminente no sexo masculino, contrastando com os resultados deste estudo. 
Para o MEEM, os homens apresentaram melhor desempenho e, portanto, melhores escores do que as mulheres avaliadas. A menor idade, melhor escolaridade e percepção da saúde podem influenciar neste resultado, apesar de este trabalho não ter encontrado diferenças estatisticamente significativas. O estudo do Projeto Bambuí, de Valle et al. ${ }^{28}$ concorda com o contexto supracitado, ao relatar que o gênero, a idade e a escolaridade são fatores consistentemente associados ao declínio cognitivo, apresentando estreita relação com o baixo desempenho no MEEM. Merecem destaque também os achados de Ávila-Funes et al., ${ }^{29}$ que em pesquisa sobre a validade dos déficits cognitivos como preditores de fragilidade, identificaram como indivíduos não-frágeis e que não tinham fortes evidências de déficits cognitivos aqueles que eram mais jovens, provavelmente do sexo masculino, mais instruídos e com melhor percepção da saúde autorrelatada. Há ainda, o estudo de Mongil et al. ${ }^{30}$ que por sua vez demonstraram que as mulheres institucionalizadas têm $65 \%$ de risco a mais que os homens para desenvolver demência.

Através da análise de correlação de Pearson, observou-se estreita relação entre o tempo de asilamento e a força de preensão manual, o que pode ser explicado pelo fato de a institucionalização acelerar a velocidade das perdas cognitivas e funcionais dos idosos, contribuindo para a progressão da fragilidade e, consequentemente, para o declínio de suas funções físicas, como por exemplo, da força de preensão. Segundo Rebellato et al., ${ }^{31}$ a fraqueza muscular dos idosos institucionalizados está associada a suas perdas funcionais, dependência de cuidadores, sedentarismo e aceleração do envelhecimento fisiológico. Do mesmo modo, Araújo \& Ceolim ${ }^{32}$ citam como impactos decorrentes da transferência de um idoso de sua casa para um asilo: depressão, isolamento, inatividade e dependência. Esta última é muitas vezes estimulada pelos próprios funcionários e cuidadores das instituições, que na maioria das vezes preferem fazer as atividades pelo idoso ou auxiliá-los maximamente em suas tarefas, estimulando o declínio funcional desses indivíduos, que por mais que já apresentem inabilidade para executar tarefas simples, nem sempre são incapazes de fazê-las. Diante deste quadro de inatividade, aparecem inúmeros danos motores caracterizados por alterações da força muscular, da amplitude de movimento e da mobilidade.

Também houve correlação $(p<0,05)$ entre a função cognitiva, massa corporal e altura com a força de preensão. As interrelações entre função física e cognitiva são bem documentadas, e alguns pesquisadores enfatizam que indivíduos com alterações no sistema motor e diminuição na força de preensão têm maior risco de declínio cognitivo e demência. ${ }^{29}$ Os déficits cognitivos são progressivos e cumulativos com o processo de senilidade/senescência, levando os idosos a diminuírem cada vez mais a frequência de suas atividades físicas, imprescindíveis para a manutenção da massa e força muscular. Neste contexto, o impacto decorrente da inatividade pode chegar a ponto de comprometer totalmente as AVDs, resultando em invalidez e dependência. ${ }^{33}$

Em relação à massa e a altura, deve ser mencionada a influência direta dos fatores antropométricos, em especial do índice de massa corporal (IMC), altamente associado com a taxa de gordura e, portanto, com o declínio da aptidão física sobre as limitações funcionais. ${ }^{34} \mathrm{~A}$ correlação entre características antropométricas e força de preensão era esperada, já que vem sendo apontada em pesquisas similares. ${ }^{35}$

No tocante à velocidade de marcha, foram encontradas correlações positivas com o nível cognitivo e com o desempenho nas atividades instrumentais da vida diária (AIVDs). Verifica-se a concordância destes dados com os resultados do trabalho de Dirik et al., ${ }^{12}$ que mostraram relação entre déficits mentais em idosos institucionalizados com diminuição tanto do nível funcional quanto da mobilidade. Em contrapartida, Oliveira et al. ${ }^{36}$ não encontraram correlação entre as alterações cognitivas detectadas pelo MEEM com o desempenho da mobilidade 
em idosos institucionalizados, relatando que pacientes com declínios cognitivos importantes ou até mesmo demência não necessariamente terão diminuição da mobilidade. Porém, nesse mesmo estudo, os autores ressaltam a importância da mobilidade e deslocamento no ambiente para a independência na realização das AVDs, encontrando uma associação entre mobilidade e independência funcional, o que corrobora nossos dados.

O teste $t$ não mostrou diferenças nas médias $\mathrm{da}$ força de preensão e velocidade de marcha em relação a nenhuma doença crônica autorrelatada. Talvez, as medicações de uso contínuo e o acompanhamento médico frequente no grupo de idosos institucionalizados tenham contribuído para o controle das doenças avaliadas, que por sua vez não influenciaram nas variáveis mensuradas. Além disso, outros parâmetros, que não o autorrelato de doenças crônicas podem ser mais fidedignos para medir o nível de saúde na população geriátrica, como por exemplo, o fenótipo de fragilidade e a capacidade funcional, bastante estudados nos dias atuais.

Algumas possíveis limitações desta pesquisa devem ser consideradas, como o pequeno tamanho amostral $(n=20)$ e a seleção da amostra por conveniência e não de forma randomizada. Tais fatores podem limitar a validade externa dos resultados. Apesar disso, a relevância de trabalhos como este deve ser mencionada, pois partindo do pressuposto de que idosos institucionalizados

\section{REFERÊNCIAS}

1. Garrido R, Menezes PR. O Brasil está envelhecendo: boas e más notícias por uma perspectiva epidemiológica. Rev Bras Psiquiatr 2002; 24: 3-6.

2. Nasri F. O envelhecimento populacional no Brasil. Einstein 2008; 6 (1):S4-S6.

3. Lima-Costa MF, Veras RP. Saúde pública e envelhecimento. Cad Saúde Pública 2003; 19:700-1.

4. Fried LP, Tangen CM, Walston J, Newman AB, Hirsch C, Gottdiener J, et al. Frailty in older adults: evidence for a phenotype. J Gerontol 2001; 56A(3):146-56. estão mais propensos a se tornarem frágeis ou mesmo a agravarem esta condição, os dados obtidos através deste tipo de pesquisa permitem traçar um perfil das condições gerais de saúde e funcionalidade desses indivíduos. Isso pode ser essencial para auxiliar em diagnósticos precoces da síndrome da fragilidade e, portanto, para prevenir e retardar agravos à saúde.

\section{CONCLUSÃO}

Levando em consideração todo o exposto, conclui-se que os idosos institucionalizados são mais vulneráveis à síndrome da fragilidade, enfrentando, portanto, maiores perdas funcionais, como as alterações na força de preensão manual e velocidade de marcha, além de perdas cognitivas, observadas pelos baixos escores no MEEM. Sendo assim, torna-se necessária uma atenção redobrada e dentro de um contexto multiprofissional a esta parcela da população, pois todas as limitações desenvolvidas, somadas à tristeza e ao isolamento frequentes no ambiente asilar, podem ser decisivas para a progressão da debilidade e morte. Desta forma, investigações aprofundadas dos problemas mais comuns enfrentados por idosos asilados são imprescindíveis para o desenvolvimento de estratégias de intervenção voltadas para as necessidades prioritárias, atuando na melhoria funcional e da qualidade de vida.
5. Bandeen-Roche K, Xue QL, Ferrucci L, Walston J, Guralnik JM, Chaves P, et al. Phenotype of frailty: characterization in the women's health and aging studies. J Gerontol 2006; 16: 262-6.

6. Fried LP, Walston JM. Frailty and failure to thrive. In: Hazard WR, Blass JP, Ettinger WH Jr, Halter JB, Ouslander J ,editors. Principles of Geriatric Medicine and Gerontology. 5th ed. New York: McGraw-Hill; 2003.

7. Macêdo C, Gazzola JM.; Najas M. Síndrome da fragilidade no idoso: importância da fisioterapia. Arquivos brasileiros de ciências da saúde 2008; 33(3):177-84. 
8. Montenegro SMRS, Silva CAB. Os efeitos de um programa de fisioterapia como promotor de saúde na capacidade funcional de mulheres idosas institucionalizadas. Revista brasileira de geriatria e gerontologia 2007; 10(2).

9. Bortz WM. A conceptual framework of frailty: a review. Gerontol 2002; 57: 283-8.

10. Vivan AS, Argimon IIL. Estratégias de enfrentamento, dificuldades funcionais e fatores associados em idosos institucionalizados. Cad Saúde Pública 2009; 25(2):436-44.

11. Cuesta VH, Acosta VH, Benito LO, Maderos FI. Prevalencia y factores de riesgo del trastorno de la memoria asociado a la edad en un área de salud. Rev Neurol 2006; 46(3):137-42.

12. Dirik A, Cavlak U, Akdag B. Identifying the relationship among mental status, functional independence and mobility level in turkish institutionalized elderly: gender differences. Arch Gerontol Geriatr 2006; 42(3):339-50.

13. Vilas Boas RF. A capacidade funcional e o nível de aptidão física para realização de atividades de vida diária em um grupo de idosos [dissertação]. Franca: Universidade de Franca; 2005.

14. Folstein MF, Folstein SE, McHugh PR. Minimental state: a practical method for grading the cognitive state of patients for clinician. J Psychiatr Res1975; 12: 189-98.

15. Almeida MF, Barata RB, Monteiro CV, Silva ZP. Prevalência de doenças crônicas auto-referidas e utilização de serviços de saúde, PNAD/1998, Brasil. Ciên Saúde Colet 2002;7:743-56.

16. Lawton MP, Brody EM. Assessment of older people: self-maintaining and instrumental activities of daily living. Gerontologist 1969; 9:179-86.

17. Katz S, Ford AB, Moskowitz RW, Jackson BA, Jaffe MW. Studies of illness in the aged. The index of ADL: a standardized measure of biological and psychosocial function. JAMA 1963;185:914-9.

18. Radloff LS. The CES-D scale: a self-report depression scale for research in the general population. Applied psychological measurement 1977;1:385-401.

19. Brucki SMD, Nitrini R, Caramelli P, Bertolucci PHF, Okamoto IH . Normas sugeridas para o uso do Mini-Exame do Estado Mental no Brasil. Arq Neuropsiquiatr 2003;61(3B):777-8.

20. Brasil, Ministério da Saúde. Resolução 196/96 do Conselho Nacional de Saúde/MS sobre Diretrizes e Normas Reguladoras de Pesquisa envolvendo seres humanos. Diário Oficial da União, 10 de outubro de 1996

21. Pavan FJ, Meneghel SN, Junges JR. Mulheres idosas enfrentando a institucionalização. Cad Saúde Pública 2008; 24(9):2187-9.

22. Converso MER, Iartelli I. Caracterização e análise do estado mental e funcional de idosos institucionalizados em instituições públicas de longa permanência. J Bras Psiquiatr 2007; 56(4):267-72.

23. Valentine RJ, McAuley E, Vieira VJ, Baynard T, $\mathrm{Hu} \mathrm{L}$, Evans EM, et al. Sex differences in the relationship between obesity, C-reactive protein, physical activity, depreession, sleep quality and fatigue in older adults. Brain Behav Immun. 2009 Jul;23(5):643-8.

24. Bautmans I, Gorus E, Njemini R, Mets T. Handgrip performance in relation to selfperceived fatigue, physical functioning and circulating IL-6 in elderly personswithout Inflammation. BMC Geriatr 2007; 7:5.

25. Moreh E, Jacobs JM, Stessman J. Feeling tired predicts functional status, physical activity, and mortality in elderly people. J Am Geriatr Soc 2009 Apr;57(4):742-3.

26. Frith J, Jones D, Newton JL. Chronic liver disease in an ageing population. Age and Ageing. 2009; 38:11-8.

27. Dey DK, Bosaeus I, Lissner L, Steen B. Changes in body composition and its relation to muscle strength in 75-year-old men and women: a 5-year prospective follow-up study of the NORA cohort in Göteborg, Sweden. Nutrition 2009; 25:613-9.

28. Valle EA, Castro-Costa E, Firmo JOA, Uchoa E, Lima-Costa MF. Estudo de base populacional dos fatores associados ao desempenho no mini exame do estado mental entre idosos: Projeto Bambuí. Cad Saúde Pública 2009; 25(4):918-26.

29. Ávila-Funes JA, Amieva H, Barberger-Gateau P, Le Goff M, Raoux N, Ritchie K, et al. Cognitive impairment improves the predictive validity of the phenotype of frailty for adverse health outcomes:the three-city study. J Am Geriatr Soc 2009 Mar;57(3):453-61

30. Mongil RL, Trigo JAL, Sanz FJC, Gómez ST, Colombo TL. Prevalencia de demencia en pacientes institucionalizados: estudio RESYDEM. Rev Esp Geriatr Gerontol 2009; 44(1):5-1.

31. Rebelatto JR, Castro AP, Chan A. Quedas em idosos institucionalizados: características gerais, fatores determinantes e relações com a 
força de preensão manual. Acta ortopedica brasileira 2007; 15 (3):151-4.

32. Araújo MOPH, Ceolim MF. Avaliação do grau de independência de idosos residentes em instituições de longa permanência. Rev Esc Enferm USP 2007; 41(3):378-85.

33. Raji MA, Kuo YF, Snih SA, Markides KS, Peek MK, Ottenbacher KJ. Cognitive status, muscle strength, and subsequent disability in older mexican americans. J Am Geriatr Soc 2005; 53(9):1462-8.

34. Arroyo P, Lera L, Sánchez H, Bunout D, Santos JL, Albala C. Indicadores antropométricos, composición corporal y limitaciones funcionales en ancianos. Rev Med Chil 2007; 135: 846-54.

35. Geraldes AAR, Oliveira ARM, Albuquerque RB, Carvalho JM, Farinatti PT. A Força de preensão manual é boa preditora do desempenho funcional de idosos frágeis: um estudo correlacional múltiplo. Revista brasileira de medicina do esporte 2008; 14(1).

36. Oliveira DLC, Goretti LC, Pereira LSM. O desempenho de idosos institucionalizados com alterações cognitivas em atividades de vida diária e mobilidade: estudo piloto. Rev Bras Fisioter 2006; 10(1): 91-6. 
